\title{
Unsusual manifestation of terra firme-forme dermatosis - upper eyelid and orbital rim
}

\section{Uwe Wollina}

\begin{abstract}
Department of Dermatology and Allergology, Academic Teaching Hospital Dresden, Friedrichstrasse 41, 01067 Dresden, Germany
\end{abstract}

Corresponding author: Prof. Uwe Wollina, E-mail: wollina-uw@khdf.de

\begin{abstract}
Terra firme-forme dermatosis is a benign exogenous dermatosis found in all age-groups. The typical localizations include neck, face, trunk and ankles. Here we report on an 88-year-old male with a medical history of stroke and paraplegia of the legs who presented with asymptomatic, hyperkeratotic and slightly hyperpigmented lesions on his upper lids. Wiping of skin with alcohol pads completely removed the lesions confirming the diagnosis of terra firmeforme dermatosis of upper eyelids. Although affection of lower eyelids has reported before, the localization on upper eyelids only is very unusual.
\end{abstract}

Key words: Terra firme-forme dermatosis; Dirty dermatoses; Skin care; Hygiene; Dermatosis negelecta

\section{INTRODUCTION}

Terra firme-forme dermatosis is a benign skin disorder also known as dermatitis neglecta, unwashed dermatosis or Duncan's dirty dermatosis [1-3]. It is characterized by asymptomatic dirt-like plaques with typically localization on neck, face, trunk and ankles [4-6]. Its etiology is not completely understood. Retention of keratinocytes in combination of sebum, residues of ointments and dirt contribute to hyperpigmented lamellar and partially whorled orthohyperkeratosis [1]. All ages can be affected [3,7].

\section{CASE REPORT}

An 88-year-old male patient presented to the department for surgical correction of severe phimosis and secondary balanophosthitis. His medical history was positive for stroke and paraplegia of the legs. He lived in a nursery.

By clinical examination hyperkeratotic, slightly hyperpigmented plaques and scales were observed on his upper lids and orbital rim (Fig. 1). There was no pruritus or any other subjective symptom. There were no clinical signs of seborrheic dermatitis or tinea. The lesions could easily be removed by wiping with alcohol pads. The diagnosis of terra firme-forme dermatosis was confirmed.

\section{DISCUSSION}

Terra firme-forme dermatosis is an often overlooked, asymptomatic skin condition related to suboptimal hygiene and skin care $[5,6,8]$. Diagnosis is established by medical history, clinical examination and skin modified by alcohol rubbing test (SMART). SMART is performed using $70 \%$ isopropyl alcohol for wiping the skin. The commonly affected body parts are neck, face, trunk and ankles [4-6]. Eyelids are uncommonly involved. Panda et al. described three female cases aged 58 to 62 years with lower lid and periorbital presentation of terra firme-forme dermatosis after cataract surgery. Cleansing of skin was omitted for several weeks due to wound dressings. The lesions could be easily removed by soap, water or acetone [9].

Here, we describe the rare upper lid affection of an 88-year-old male patient with a positive SMART test. Because of the localization, other dermatoses need to be considered such as atopic and seborrheic dermatitis, contact dermatitis, psoriasis, rosacea and tinea [10]. 


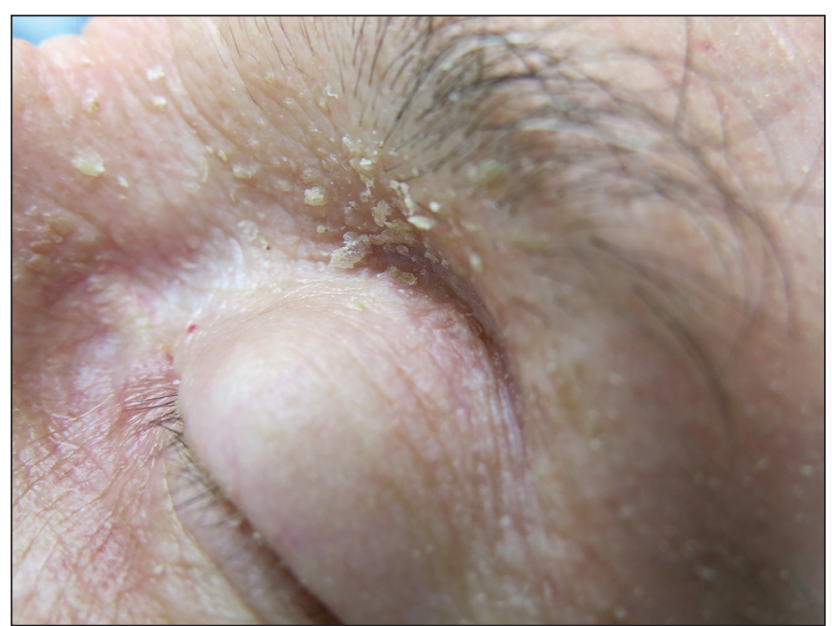

Figure 1: Terra firme-forme dermatosis of upper lid and orbital rim.

Although a rare localization, upper lids and orbital rim can be affected by terra firme-forme dermatosis. SMART test is an easy way to exclude other possible differential diagnoses.

\section{CONSENT}

The examination of the patient was conducted according to the Declaration of Helsinki principles.

\section{REFERENCES}

1. Duncan WC, Tschen JA, Knox JM. Terra firme-forme dermatosis. Arch Dermatol. 1987;123:567-9.

2. Poskitt L, Wayte J. Dermatitis neglecta: unwashed dermatosis. Br J Dermatol. 1995;132:827-9.

3. Ashique KT, Kaliyadan F, Goyal T. Terra firme-forme dermatosis: report of a series of 11 cases and a brief review of the literature. Int J Dermatol. 2016;55:769-74.

4. Greywal T, Cohen PR. Terra firme-forme dermatosis: A report of ten individuals with Duncan's dirty dermatosis and literature review. Dermatol Pract Concept. 2015;5:29-33.

5. Bharti R. Terra firma forme dermatosis and plica neuropathica - case report. Our Dermatol Online. 2017;8:474-6.

6. Babu AR, Vijayashankar M. Terra firme-forme dermatosis. Our Dermatol Online. 2013;4:89-90.

7. Unal E, Guarneri C, Chokoeva AA, Wollina U, Tchernev G. Terra firme-forme dermatosis. Wien Med Wochenschr. 2017;167:66-9.

8. Saha A, Seth J, Bindal A, Samanta AB, Gorai S, Sharma A. Dermatosis neglecta: An increasingly recognized entity with review of literature. Indian J Dermatol. 2016;61:450-2.

9. Panda M, Patro N, Samant S, Arora S. Dermatitis neglecta as a complication after cataract surgery. Indian J Ophthalmol. 2016;64:231-2.

10. Wolf R, Orion E, Tüzün Y. Periorbital (eyelid) dermatides. Clin Dermatol. 2014;32:131-40.
Copyright by Uwe Wollina. This is an open access article distributed under the terms of the Creative Commons Attribution License, which permits unrestricted use, distribution, and reproduction in any medium, provided the original author and source are credited.

Source of Support: Nil, Conflict of Interest: None declared. 